\title{
SPIRAL LIKE INTEGRAL OPERATORS
}

\author{
SHYAM K. BAJPAI \\ Departamento de Matematica \\ Universidade de Brasilia \\ Instituto de Ciencias Exactas \\ 70910 Brasilia-DF-BRASIL \\ (Received January 9, 1980)
}

ABSTRACT. In this paper we investigate the Robertson-Libera integral operators for the class of spiral like univalent and analytic functions. We find that special types of transformations preserve the class property. Our results generalize or sharpen the results recently obtained by Miller et al [7] and by Causey and White [3].

KEY WORDS AND PHRASES: Univalent analytic functions, Starlike; convex. 1980 MATHEMATICS SUBJECT CLASSIFICATION CODES: Primary 30A32, Secondary $30 A 36$.

1. INTRODUCTION.

Let $R^{+}=\{x \mid x \geq 0\}, D=\{z|| z \mid<1\}, C=$ the complex numbers,

$S=\left\{f \mid f: D \rightarrow C, f\right.$ to be regular, univalent and $\left.f(0)=0, f^{\prime}(0)=1\right\}$,

$K_{\beta}=\left\{f \mid f \varepsilon S\right.$ and $\left.\operatorname{Re}\left\{1+\frac{z f^{\prime \prime}(z)}{f^{\prime}(z)}\right\}>\beta ; 0 \leq \beta<1\right\}, S(\alpha, \beta)=\{f \mid f \varepsilon S$, 
$\left.\operatorname{Re}\left\{e^{i \alpha} \frac{z f^{\prime}(z)}{f(z)}\right\}>\beta \cos \alpha, 0 \leq \beta<1, \frac{-\pi}{2} \leq \alpha \leq \frac{\pi}{2}\right\}, s_{\beta}^{*}=s(0, \beta), s^{*}=s(0,0)$, $S(\alpha, 0)=S(\alpha)$ and finally $I^{+}=\{n \mid n=1,2,3, \ldots\}$. $f$ is said to be starlike of order $\beta$ with respect to the origin if and only if $f \varepsilon s_{\beta}^{*}$ and $f$ is $\alpha$-spiral like of order $\beta$ if and only if $f \varepsilon S(\alpha, \beta)$.

In 1964, Robertson [10], proved the following

THEOREM A: - Let $k(z)$ denote the Koebe function $z(1-z)^{-2}$, which is univalent and starlike with respect to the origin for $|z|<1$. Then the function

$$
S(z)=\frac{2}{2} \int_{0}^{z} k(t) d t
$$

is also univalent and starlike with respect to the origin for $|z|<1$. The path of integration is restricted in the obvious way.

THEOREM B: - Let $S(z)$ and $k(z)$ be defined as in theorem $A$. Then

$$
T(z)=[k(z)-S(z)]^{1 / 2}
$$

is univalent and starlike in $D$ and satisfies the inequality

$$
|T(z)| \leq\left(\frac{1-|z|}{|1-z|}\right) T(|z|)=[2 k(z)]^{1 / 2} \text {. }
$$

Libera [6] established that theorem $A$ holds true when $k(z)$ is replaced by any $f \varepsilon s^{\star}$. Bernardi [2] greatly generalised Libera's result. Many authors studied the operators of the form

$$
F_{f}(z)=\frac{(1+\gamma)}{z} \int_{0}^{z} t^{\gamma-1} f(t) d t
$$

where $\gamma$ is a real constant and $f$ belongs to some favoured class of functions from S (see, ref. [1] $-[14]$ ). Recently, operators (1.4) have been studied in more 
general form by Causey and White [3] and Miller, Mocanu and Reade [7], independently.

THFOREM C [3]. Suppose $f \in S^{*}, g \varepsilon \bar{K}, \alpha, c \in I^{+}, \gamma, \delta \in R^{+}$and $(2 \delta+\gamma) \leq \min (2 \alpha, 2 c)$. Then the function $F$ defined by

$$
F(z)=\left[c z^{\alpha-c} \int_{0}^{z} t^{c-1}\left(\frac{f(t)}{t}\right) \delta\left(\frac{g(t)}{t}\right)^{\gamma} d t\right]^{1 / \alpha},
$$

belongs to $\mathrm{s}^{*}$.

THEOREM D ( $[7], \underline{\text { THM } 6,}, \underline{P}, 165)$ : - Let $\xi, \beta^{*}, \gamma, \rho$ and $\delta$ be real constants satisfying the conditions $\xi \geq 0, B^{*}>0, \xi+\delta=\beta^{*}+\gamma>0$ and

$$
0 \leq \frac{\rho}{2} \leq\left\{\begin{array}{l}
\delta \quad \text { if } \quad \gamma \leq 0 \\
\min \left\{\delta, \delta-\gamma+\frac{1}{2} \min \left(\frac{\beta^{*}}{\gamma}, \frac{\gamma}{\beta^{\star}}\right)\right\} \text { if } \gamma>0 .
\end{array}\right.
$$

If $f \varepsilon s^{*}, g \varepsilon \bar{k}$ then the function

$$
F(z)=\left[\frac{\beta^{*}+\gamma}{z^{\gamma}} \int_{0}^{z} f^{\xi}(t) g^{\rho}(t) t^{\delta-\rho-1} d t\right]^{1 / \beta^{*}}
$$

belongs to $\mathrm{s}^{*}$.

Theorem C is more or less contained in Theorem D. The proof of Theorem C, due to Causey and White depends on a result of Sakaguchi $([11], p .74)$, whereas the proof of Theorem $D$, depends on a lemma due to Lewandowski, Miller and zlotkiewicz $([5], p .114)$. Since this latter lemma is more powerful than the result of Sakaguchi ( $[11], p .74)$, Theorem $D$ is more general than that of Theorem C. In this paper we shall prove certain theorems which in a way are sharper than Theorems $C$ and $D$, in the process generalizing the main theorem of Miller, Mocanu and Reade [7]: 
THEOREM E: - Let $\psi(z)=1+\ldots, \phi(z)=1+\ldots$, be functions defined in $D$ with the property $\phi(z) \psi(z) \neq 0$ there. Let $\alpha, \beta, \gamma, \delta$ be real constants such that $\alpha \geq 0, \beta>0, \delta \geq 0, \alpha+\delta>0$ and $\alpha+\delta=\beta+\gamma$. If there exists a non-negative constant $J$ that satisfies

$$
\mathrm{J} \geq \gamma+\operatorname{Re}\left\{\frac{z \psi^{\prime}(z)}{\psi(z)}\right\}, \beta+\gamma>\mathrm{J} \text { and } \delta+\operatorname{Re}\left\{\frac{z \phi^{\prime}(z)}{\phi(z)}\right\} \geq \max [0, J-\lambda(J)]
$$

where $\lambda(J)=\frac{1}{2} \max [(\beta+\gamma-J) / J, J /(\beta+\gamma-J)], \lambda(0)=0$ and if $f \varepsilon s^{*}$, then there exists a function

$$
\left.F(z)=\frac{[\beta+\gamma}{z^{\gamma} \psi(z)} \int_{0}^{z} f^{\alpha}(t) \phi(t) t^{\delta-1} d t\right]^{1 / \beta} \varepsilon s^{*}
$$

Our generalizations of these theorems depend on the following result of Jack [4] which is also due to Suffridge [14].

LEMMA A: - Suppose that $w$ is analytic for $|z| \leq r<1, w(0)=0$ and

$$
\begin{aligned}
& \left|w\left(z_{1}\right)\right|=\max _{|z|=r}|w(z)| \text {. Then } \\
& \qquad z_{1} w^{\prime}\left(z_{1}\right)=K w\left(z_{1}\right) \text { and } k \geq 1 .
\end{aligned}
$$

Ways of generalizing Theorem A have been obtained in $([3], p .458)$ and $([7], p .157)$. We shall investigate generalizing Theorem B similarly. Perhaps these generalizations of Theorem $B$ have not been investigated because application of Sakaguchi's results is not easy. However the lemma of Jack is easy and powerful enough to apply in various situations.

\section{FUNDAMENTAL THEOREMS}

Our generalization of theorems A, C, and D is the following. 
THEOREM 1: - Let $f_{n} \varepsilon S\left(\alpha, \beta_{n}\right), n=0,1,2, \ldots,\left(f_{0}=f, \beta_{0}=\beta\right)$,
$-\frac{\pi}{2} \leq \alpha \leq \frac{\pi}{2}, 0 \leq \beta_{n}<1, a, b, \lambda_{n} \varepsilon R^{+}$and $\left\{-b \beta+\sum_{n=1}^{M} \lambda_{n}\left(1-\beta_{n}\right)\right\} \cos \alpha \leq \min \{\operatorname{Re} c$ $a \cos \alpha\}$. Then the function

$$
F(z)=\left[\left(\frac{c+b e^{i \alpha}}{c-a e^{i \alpha}}\right) \int_{0}^{z} t^{c-1}[f(t)]^{b e^{i \alpha}}\left\{\prod_{n=1}^{M}\left(\frac{f_{n}(t)}{t}\right)^{\lambda_{n} e^{i \alpha}}\right\} d t\right]^{\left(\frac{e^{-i \alpha}}{a+b}\right)}
$$

belongs to $S\left(\alpha, \frac{a+b \beta-\sum_{n=1}^{M} \lambda_{n}\left(1-\beta_{n}\right)}{a+b}\right)$. In (2.1) all powers are principal ones.

PROOF OF THEOREM 1: - Let us write

$$
\begin{gathered}
G(t)=t^{c-1}[f(t)] b e^{i \alpha}\left\{\prod_{n=1}^{M}\left(\frac{f_{n}(t)}{t}\right) \lambda_{n} e^{i \alpha}\right\} \\
e^{i \alpha \frac{z F^{\prime}(z)}{F(z)}}=\left(\frac{m \cos \alpha}{a+b}\right)+i \sin \alpha+\left(\frac{1+w(z)}{1-w(z)}\right)\left(\frac{a+b-m}{a+b}\right) \cos \alpha
\end{gathered}
$$

where

$$
m=b B+a-\sum_{n=1}^{M} \lambda_{n}\left(1-\beta_{n}\right)
$$

Then by (2.1) via differentiation, we have

$$
\begin{gathered}
z^{c-a e^{i \alpha}-1}[F(z)]^{(a+b) e^{i \alpha}}\left\{\left(c-a e^{i \alpha}\right)+(a+b) e^{i \alpha} \frac{z F^{\prime}(z)}{F(z)}\right\} \\
=\left(c+b e^{i \alpha g(z) .}\right.
\end{gathered}
$$

By (2.3), (2.5) takes the following form.

$$
\begin{aligned}
& c-a e^{i \alpha}-1+(a+b) e^{i \alpha} \frac{z F^{\prime}(z)}{F(z)}+\frac{z w^{\prime}(z)}{1-w(z)}+ \\
& \frac{\left(2 a \cos \alpha-2 m \cos \alpha-c+b e^{-i \alpha}\right) z w^{\prime}(z)}{\left(c+b e^{i \alpha}\right)+\left[2 a \cos \alpha-2 m \cos \alpha-c+b e^{-i \alpha}\right] w(z)}=c-1-
\end{aligned}
$$




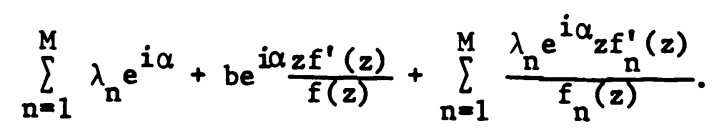

Hence

$$
\begin{aligned}
& \quad b \operatorname{Re}\left\{\frac{z e^{i \alpha_{f^{\prime}}(z)}}{f(z)}\right\}=-a \cos \alpha+(a+b) \operatorname{Re}\left\{\frac{e^{i \alpha} z F^{\prime}(z)}{F(z)}\right\}+ \\
& +\operatorname{Re}\left\{\frac{2(a+b-m) \cos \alpha z w^{\prime}(z)}{(1-w(z))\left\{c+b e^{i \alpha+w(z)}\left[2 a \cos \alpha-2 m \cos \alpha-c+b e^{-i \alpha}\right]\right\}}\right\}+ \\
& +\sum_{n=1}^{M} \lambda_{n} \cos \alpha-\sum_{n=1}^{M} \lambda_{n} \operatorname{Re}\left\{\frac{e^{i \alpha} z f^{\prime}(z)}{f_{n}(z)}\right\} .
\end{aligned}
$$

It is clear that $F(z)$ is regular in $D$ and possesses a simple zero at the origin. Hence, without loss of generality, we may assume that $w(z)$ defined by $F(z)$ in (2.3) is regular in D. Also, it follows that $w(0)=0$. Thus, if we show that $|w(z)|<1$ in $D$ then, by subordination, it will follow that $F(z)$ is spiral like of order $\left(\frac{m}{a+b}\right)$ whenever $0 \leq \frac{m}{a+b}<1$. Suppose there existed a point $z_{1}$ in $D$ at which $\left|w\left(z_{1}\right)\right|=1$, then by Jack's lemma $z_{1} w\left(z_{1}\right)=K w^{\prime}\left(z_{1}\right), K \geq 1$. For this value of $z=z_{1}$, we find that (2.7) takes the following form

$$
\begin{gathered}
b \operatorname{Re}\left\{\frac{z_{1} f^{\prime}\left(z_{1}\right) e^{i \alpha}}{f\left(z_{1}\right)}-\beta \cos \alpha\right\} \leq \\
\leq \frac{-k(a+b-m)(R e c-a \cos \alpha+m \cos \alpha) \cos \alpha}{\left|c+b e^{i \alpha}+w(z)\left\{2 a \cos \alpha-2 m \cos \alpha-c+b e^{-i \alpha}\right\}\right|^{2}} \leq 0
\end{gathered}
$$

if $a+b-m \geq 0$ and $\operatorname{Re} c \geq(a-m) \cos \alpha$.

But, this contradicts that $f \varepsilon S(\alpha, \beta)$. Hence, we must have $|w(z)|<1$ in $D$. This completes the proof of the theorem.

Some consequences of Theorem 1 , are the following.

(A) Let $\beta_{1}=\frac{1}{2}, \lambda_{1}=\gamma, \lambda_{m}=0$, for $m \geq 2, b=\delta, \alpha=0, a+b=\beta^{*}, c+b=c$, 


$$
\begin{aligned}
& f_{1}=g \varepsilon s_{\frac{1}{2}=\beta_{1}}^{*}\left(=K_{0}\right) \text {, and } f \varepsilon s_{0}^{*} \text { then by Theorem 1, it follows that } \\
& F(z)=\left[\frac{C}{C-\beta^{*}} \int_{0}^{z} t^{C-1}\left(\frac{f(t)}{t}\right) \delta\left(\frac{g(t)}{t}\right) \gamma_{d t}\right]^{1 / \beta^{*}} \varepsilon S^{*}\left(0, \frac{2 \beta^{*}-\gamma-2 \delta}{2 \beta^{*}}\right) \text { for min }(\operatorname{Re} C, a) \geq \delta+\frac{\gamma}{2} .
\end{aligned}
$$

This is more general and sharper than the main theorem of Causey and white ( $[3]$, Thm 3.1, p. 459).

(B) Take $b=\xi_{1}, \rho=\lambda_{1}, \gamma=c-a, a+b=\beta^{\star}, \alpha=0=\beta, M=1, c+b=\xi+\delta=\beta^{*}+\gamma$, $f_{1}=g, \beta_{1}=\frac{1}{2}$ and $c \varepsilon R^{+}$. Then, Theorem $B$, follows with $F \in S^{*}\left(0, \frac{2 \beta^{*}-2 \xi-\rho}{2 \beta^{*}}\right)$ and $0 \leq \frac{\rho}{2} \leq \min (\delta, \delta-\gamma)$ if $\gamma>0$ and $0 \leq \frac{\rho}{2} \leq \delta$ if $\gamma \leq 0$. This sharpens theorem 6 of $([7], p .165)$.

(C) If we take $b=\xi, \lambda_{1}=\rho, \gamma=c-a, a+b=\beta^{*}, \alpha=0, M=1, c+b=\xi+\delta=\beta^{*}+\gamma$, $f_{1}=g, \beta=\frac{1}{2}=\beta_{1}, c \varepsilon R, f, g \varepsilon K_{\beta=0} \subset s_{1 / 2}^{*}$, then $F$ of theorem $\frac{1 \varepsilon S_{2 \beta^{*}-2 \xi-\rho}^{*}}{2 \beta^{\star}}$ whenever $-\xi \leq \rho \leq \min (2 \delta+\xi, 2 \delta+\xi-2 \gamma)$. This is a slightly improved version of theorem 7 of $[7]$ which implies that $F \varepsilon s_{0}^{*}$ and where $0 \leq \rho \leq \min \{2 \delta+\xi$, $2 \delta+\xi-2 \gamma+\frac{1}{2} \min \left(\frac{\beta^{*}}{\gamma}, \frac{\gamma}{\beta^{\star}}\right)$ if $\gamma>0$ and $0 \leq \rho \leq 2 \delta+\xi$ if $\gamma \leq 0$.

(D) If we let $b=0=\alpha, a=1, c=1=M, f_{1} \varepsilon s_{1 / 2}^{*}=s\left(0, \frac{1}{2}\right) \subseteq k_{0}$ then by theorem 1, it follows that

$$
F(z)=\int_{0}^{z}\left(\frac{f(t)}{t}\right)^{\lambda}{ }_{d t} \varepsilon S\left(0,1-\frac{\lambda}{2}\right) \text { if } \frac{\lambda}{2} \leq 1 .
$$

This is clearly an improvement of Merkes-Wright's result [8] and also to that of Miller, Mocanu and Reade ([7], p. 165).

(E) If we let $a=\lambda_{n}=0, b>0$ then it follows that 
$F(z)=\left[\frac{c+b e^{i \alpha}}{z^{c}} \int_{0}^{z} t^{c-1}[f(t)]^{b e^{i \alpha}} d t\right] \frac{1}{b e^{i \alpha}} \varepsilon S(\alpha, \beta)$ for $f \varepsilon s(\alpha, \beta)$ and $\min (\operatorname{Re} c, 0)>-b \beta$. If further $\alpha=0=\beta$ then $f \varepsilon s^{*}$ implies $F \in s^{*}$ for $\operatorname{Re} c>0$. Whenerver $c$ is real, this result is due to singh [13].

(F) If we let $a=0=\lambda_{n}$ then the function $F$ defined in (E) enables us to generalize Theorem 4 of [7]. Also, it is worth noticing that the function $F$ defined in (E) is a generalization of $\alpha$-spiral- $\beta$-convex function [12] which in turn is a generalization of alpha convex functions due to Mocanu [9].

A slightly different method than used for proof of Theorem 1, permits us to generalize Theorem $\mathrm{E}$ as follows.

THEOREM 2: - Let $\psi(z)=1+\ldots$, and $\phi(z)=1+\ldots$ be analytic in $D$ with $\phi(z) \cdot \psi(z) \neq 0$ there. Let $\alpha \geq 0, \beta>0, \operatorname{Re}(\gamma-\delta)=\alpha-\beta, \operatorname{Re} \gamma \geq 0$ and $\mathrm{f} \varepsilon \mathrm{s}^{*}$ then $\mathrm{F} \varepsilon \mathrm{s}_{\mathrm{m}_{0}}^{*}$ for some $\mathrm{m}_{\mathrm{o}}$ where

$$
\begin{aligned}
& F(z)=\left[\left(\frac{\beta+\gamma}{z^{\gamma} \psi(z)}\right) \int_{0}^{z} f^{\alpha}(t) \phi(t) t^{\delta-1} d t\right] 1 / \beta=z+\ldots, \\
& \text { and } 0 \leq m_{0}<1 . \text { Furthermore, if } m=\max _{z \in D} \frac{1}{\beta}\left\{\operatorname{Re}\left(\frac{z \psi^{\prime}(z)}{\psi(z)}\right)\right\}+m_{0}
\end{aligned}
$$

then $m$ satisfies the following inequality

$$
\begin{gathered}
{\left[\alpha+\mathrm{m} \beta-\beta-h e\left\{\frac{z \phi^{\prime}(z)}{\phi(z)}\right\}\right][|\gamma+\beta|+|\beta-2 m \beta-\gamma|]^{2} \leq} \\
2 \beta(1-\mathrm{m})(\mathrm{m} \beta+\operatorname{Re}\{\gamma\}) .
\end{gathered}
$$

iloreover, if $G(z)=F(z) \psi^{1 / \beta}(z)$, then $G \varepsilon S_{m}^{*}$ where $m$ is the largest value obtainec from $(2.10)$. 
PROOF OF THEOREM 2: - Let us write $G(z)=F(z) \psi^{1 / \beta}(z)$ and $\frac{z G^{\prime}(z)}{G(z)}=\frac{1+(1-2 m) w(z)}{1-w(z)} ; 0 \leq m<1$. Then, $w(z)$ is regular in $D, w(0)=0$. Some easy simplifications give us

$$
\begin{aligned}
& \alpha \operatorname{Re}\left\{\frac{z f^{\prime}(z)}{f(z)}\right\}=\operatorname{Re}(\gamma-\delta)+m \beta+(1-m) \operatorname{Re}\left\{\frac{1+w(z)}{1-w(z)}\right\} \beta \\
+ & \operatorname{Re}\left\{\frac{2 \beta(1-m) z w^{\prime}(z)}{(1-w(z))((\gamma+\beta)+(\beta-2 m \beta-\gamma) w(z))}\right\}-\operatorname{Re}\left\{\frac{z \phi^{\prime}(z)}{\phi(z)}\right\} .
\end{aligned}
$$

Now, if it is not possible for all $z \in D$ that $|w(z)|<1$ then there exists a $z_{1} \varepsilon D$ for which $\left|w\left(z_{1}\right)\right|=1$. Then by Jack's lemna $z_{1} w^{\prime}\left(z_{1}\right)=K w\left(z_{1}\right), K \geq 1$. For such a $z_{1}$, we have

$$
\begin{aligned}
& \alpha \operatorname{Re}\left\{\frac{z_{1} f^{\prime}\left(z_{1}\right)}{f\left(z_{1}\right)}\right\} \leq \operatorname{Re}\{\gamma-\delta\}-\operatorname{Re}\left\{\frac{z \phi^{\prime}(z)}{\phi(z)}\right\}+m \beta- \\
& \frac{2 E(1-\mathrm{m})(\mathrm{m} \beta+\operatorname{Re}(\gamma))}{\{|(\gamma+\beta)+(\beta-2 \mathrm{~m} \beta-\gamma)|\}^{2}} .
\end{aligned}
$$

The last inequality leads to a contradiction on $f \varepsilon S^{*}$ whenever $(2.10)$ is satisfied. Thus, we must have,$w(z) !<1$ in $D$ and the theorem is proved for $G \in S_{m}^{*}$ and it also fcllows that $F \in S_{m_{0}}^{*}$. A special case is of interest.

(A') Let $\operatorname{Im}(\gamma-\delta)=0$. Then $\gamma-\delta=\alpha-\beta$. In this case (2.10) possesses simple solutions. There are possibilities (i) $3-2 \mathrm{~m} \beta-\gamma \geq 0$, (ii) $\beta-2 \mathrm{~m} \beta-\gamma \leq 0$. For (i), we obtain m must satisfy $m \beta+\gamma \geq 2 \beta\left[\alpha-m \beta-\beta-\operatorname{Re}\left(\frac{z \phi^{\prime}(z)}{\phi(z)}\right)\right](1-m)$ whereas in the case (ii) it must satisfy the inequality $\beta(1-m) \geq 2(\gamma+m \beta)\left[\alpha-\beta-m \beta-\operatorname{Re}\left\{\frac{z \phi^{\prime}(z)}{\phi(z)}\right\}\right]$.

3. OTHER FUNCTIONS DEFINED BY INTEGRAL REPRE SENTATIONS

THEOREM 3: - Let $f$ be starlike and $F(z)=\frac{1+c}{z^{c}} \int_{0}^{z} t^{c-1} f(t) d t$. Then $F \in S_{m}^{*}$ 
where $m$ satisfies the inequality $m[|1+c|+|1-c-2 m|]^{2} \leq 2(1-m)(m+\operatorname{Re}\{c\})$ and $0 \leq m<1$.

PROOF: - A simple calculation shows that

$$
\left[c+\frac{z F^{\prime}(z)}{F(z)}\right] F(z)=(1+c) f(z) .
$$

If possible, let us write

$$
\frac{z F^{\prime}(z)}{F(z)}=(1-m)\left(\frac{1+w(z)}{1-w(z)}\right)+m ; \quad 0 \leq m<1 .
$$

Then (3.1) and (3.2) gives us,

$$
\begin{gathered}
\frac{z f^{\prime}(z)}{f(z)}=m+(1-m)\left(\frac{1+w(z)}{1-w(z)}\right) \\
+\frac{2(1-m) z w^{\prime}(z)}{[1-w(z)][(1+c)+(1-c-2 m) w(z)]} .
\end{gathered}
$$

From (3.2), it follows that $w(0)=0, w(z)$ is regular in $D$, if it were not possible that $|w(z)|<1$ in $D$ then there exists $z_{1} \varepsilon D$ such that $\left|w\left(z_{1}\right)\right|=1$ and by Jack's lemma $z_{1} w^{\prime}\left(z_{1}\right)=K w\left(z_{1}\right)$ for some $K \geq 1$. Hence at $z=z_{1}$, by (3.3), we get

$$
\begin{aligned}
\operatorname{Re}\left\{\frac{z_{1} f^{\prime}\left(z_{1}\right)}{f\left(z_{1}\right)}\right\} & =m+\frac{2(1-m) K R e\left\{\left(w\left(z_{1}\right)-1\right)\left((1+\bar{c})+(1-\bar{c}-2 m) \bar{w}\left(z_{1}\right)\right\}\right.}{\left|\left(1-w\left(z_{1}\right)\right)\right|^{2}|(1+c)+(1-c-2 m) w(z)|^{2}} \\
& =m-\frac{2 K(1-m)\left(m+c_{1}\right)}{\left|(1+c)+(1-c-2 m) w\left(z_{1}\right)\right|^{2}} ; c_{1}=\operatorname{Re}\{c\} \\
& \leq m-\frac{2(1-m)\left(m+c_{1}\right)}{\left|(1+c)+(1-c-2 m) w\left(z_{1}\right)\right|^{2}} ; c_{1} \geq-m \\
& \leq m-\frac{2(1-m)\left(m+c_{1}\right)}{(|1+c|+|1-c-2 m|)^{2}} ; \quad c_{1} \geq-m .
\end{aligned}
$$

But, then right hand side is $\leq 0$. Hence, we have a contradiction for $f \in s_{0}^{*}$. Thus, we must have $|w(z)|<1$ and Theorem 3 is proved. 
As a consequence if we let $c=1$, then $F(z)=\frac{2}{z} \int_{0}^{z} f(t) d t, f \varepsilon s^{*}$, is starlike of order $m$ where $m$ is the largest possible positive value satisfying the inequality $2\left[\frac{\sqrt{17}-3}{4}-m\right]\left[\mathrm{m}+\frac{\sqrt{17}+3}{4}\right] \geq 0$. Hence, we must have $\mathrm{m}=\frac{\sqrt{17-3}}{4}$. Thus, we have recovered by our method, a result of Miller, Mocanu and Reade ([7], pp. 162-163), an improvement of a theorem of Libera [6]. Similarly, if we admit $c$ to be real number such that $c+m \geq 0$ and $m$ is the largest positive value satisfying the inequality $m \leq 2(1-m)(m+c) /\{|1+c|+|c+2 m-1|\}^{2}$ then by Theorem 3 , we obtain that for $f \varepsilon S^{*}$ the function $\left.F(z)=(1+c) / z^{c}\right) \int_{0}^{z} t^{c-1} f(t) d t \varepsilon S_{m}^{*}$. It is easy to see that $m \geq \frac{1-c}{2}$ and hence, we must have $m=\left\{-(2 c+1)+\sqrt{(2 c-1)^{2}+8(1+c)}\right\} / 4$. This is another, result of Miller, Mocanu and Reade ([7], pp. 162-163) obtained by our method.

THEOREM 4: - If $f \varepsilon S^{*}, \alpha \geq 0$ and $F(z)=\left[z^{\beta-1} \int_{0}^{z}[f(t) / t]^{\alpha} d t\right]^{1 / \beta}, 0 \leq \beta \leq 1$ then $F \in S_{m}^{*}$ where $m$ satisfies the inequality

$$
(\alpha-\beta+m \beta)[1+|2 \beta-2 m \beta-1|]^{2} \leq 2 \beta(1-m)[1-\beta(1-m)]
$$

PROOF: - If possible, let $\frac{z F^{\prime}(z)}{F(z)}=(1-m)\left(\frac{1+w(z)}{1-w(z)}\right)+m=\frac{1+(1-2 m) w(z)}{1-w(z)}, 0 \leq m<1$. Then, we obtain

$$
\begin{gathered}
\alpha \operatorname{Re}\left\{\frac{z f^{\prime}(z)}{f(z)}\right\}=\alpha-\beta+m \beta+\beta(1-m) \operatorname{Re}\left\{\frac{1+w(z)}{1-w(z)}\right\}+ \\
\operatorname{Re}\left\{\frac{2 \beta z w^{\prime}(z)(1-m)}{[1-w(z)][1+(2 \beta-2 m \beta-1) w(z)]}\right\} .
\end{gathered}
$$

Clearly, we have $w(0)=0, w(z)$ is regular in $D$ and if it is not possible $|w(z)|<1$ in $D$ then there exists a $z_{1} \varepsilon D$ such that $\left|w\left(z_{1}\right)\right|=1$ and by Jack's lemma $z_{1} w^{\prime}\left(z_{1}\right)=K w\left(z_{1}\right) ; K \geq 1$. Then, for this value of $z_{1}$, we get 


$$
\alpha \operatorname{Re}\left\{\frac{z_{1} f^{\prime}\left(z_{1}\right)}{f\left(z_{1}\right)}\right\} \leq \alpha-\beta+m \beta-\frac{2 \beta(1-m)(1+m k-\beta)}{\{1+|2 \beta-2 m \beta-1|\}^{2}} .
$$

This contradicts that $f \in S^{*}$. Hence, we must have $|w(z)|<1$ and so proof is finished. This improves Theorem 3 of [7].

THEOREM 5: - If $f \varepsilon S^{*}$ then $F(z)=\int_{0}^{z}\left[\frac{f(t)}{t}\right]^{\alpha} d t \varepsilon s_{m}^{*}$ for $0 \leq \alpha \leq 1$ and $\mathrm{m}=\frac{(1-2 \alpha)+\sqrt{4 \alpha^{2}-4 \alpha+9}}{4}$

PROOF: - Choosing $\beta=1$, in Theorem 4, we obtain $F \varepsilon S_{m}^{*}$ if $m$ satisfies the inequality

$$
\alpha-1+m-\frac{2 m(1-m)}{[1+|1-2 m|]^{2}} \leq 0 .
$$

Now, there are two cases: (i) $m \geq \frac{1}{2}$ or (ii) $m \leq \frac{1}{2}$. If $0 \leq \alpha \leq 1$ then (3.7) is always satisfied for $m \leq \frac{1}{2}$. Hence, $m \geq \frac{1}{2}$. In this case we find, whenever

$$
x_{1}=\frac{(1-2 \alpha) \sqrt{4 \alpha^{2}-4 \alpha+9}}{4} \leq m \leq \frac{(1-2 \alpha)+\sqrt{4 \alpha^{2}-4 \alpha+9}}{4}=x_{2}
$$

the inequality (3.7) is always satisfied. But, it is also true that $x_{1} \leq \frac{1}{2} \leq x_{2}$. Hence, $m=\left[(1-2 \alpha)+\sqrt{4 \alpha^{2}-4 \alpha+9}\right] / 4$.

Finally when $\alpha>1$ the same method gives us the following improvement of a result of Silvia $[12]$.

THEOREM 6: - Let $\mathrm{f} \varepsilon \mathrm{S}, \mathrm{f}(\mathrm{z}) \mathrm{f}^{\prime}(\mathrm{z}) / \mathrm{z} \neq 0, \frac{-\pi}{2} \leq \lambda \leq \frac{\pi}{2}$ and $\operatorname{Re}\left\{\left(e^{i \lambda}-\alpha\right) \frac{z f^{\prime}(z)}{f(z)}+\alpha\left(1+\frac{z f^{\prime \prime}(z)}{f^{\prime}(z)}\right)\right\}>\beta \cos \lambda, 0 \leq \lambda \leq 1,-\infty<\alpha<\infty$. Then $f$ is $\lambda$-spiral like of order $m(>\beta)$, i.e. $f \in s(\lambda, m)$ if $\alpha \geq 1$ and $f \in s(\lambda, \beta)$ if $\alpha \leq 1$ where $m$ is the largest real number satisfying the inequality 


$$
(m-\beta)\left\{1+\left[1-4 m(1-m) \cos ^{2} \lambda\right]^{1 / 2}\right\} \leq 2 m \alpha(1-m) \cos \lambda .
$$

In special, if we take $\lambda=0=\beta$, then the class of alpha convex functions introduced by Mocanu [9] are produced if, further, $\alpha \geq 1$ then these functions are of order $m=\frac{\sqrt{8 \alpha+\alpha^{2}}-\alpha}{4}$. This is a slight improvement of a result of Miller, Mocanu and Reade [7]. We omit the proof of this theorem, which is long but straightforward.

\section{GENERALIZATION OF THEOREM B}

First of all we note that $f(z)=z \varepsilon S^{*}$ and so trivially $S(z)=\frac{2}{2} \int_{0}^{z} t d t=$ $z \varepsilon S^{*}$ but $T(z)=\left[z-\frac{2}{z} \int_{0}^{z} t d t\right]^{1 / 2} \equiv 0$, a non-univalent function. Hence, there is no chance of an exact analogue of theorem B. However, there are some variant analogues of theorem $B$.

THEOREM 7: - If $f \in K_{0}$ then $T(z)=2\left[f(z)-\frac{1}{z} \int_{0}^{z} f(t) d t\right] \varepsilon s_{m}^{*}$ where $\mathrm{m}=\left(\frac{\sqrt{17}-3}{4}\right)$.

The proof of this theorem is similar to that of Theorem 3 and just follows by assuming that $0 \leq m \leq(1-m) / 2(1+m)$ or by using theorem 3 . This is an improvement of a result of Libera $([6], p .757)$.

Theorem 7 suggests the following.

THEOREM 8: - If $f \in K_{0}$ then $F(z)=\left(\frac{e^{i \alpha}-e^{i \beta}}{z}\right)\left[\int_{0}^{z} f\left(t e^{i \alpha}\right) d t-\int_{0}^{z} f\left(t e^{i \beta}\right) d t\right] \varepsilon$ $s_{m}^{*}$ for $\alpha \notin \beta$ and $\alpha, \beta \varepsilon[0,2 \pi)$ where $m=(\sqrt{17}-3) / 4$.

The proof of this theorem depends on the following lemma: 
LEMMA 2: If $f \in \mathrm{K}_{\delta}$ then $\alpha, \beta \varepsilon[0,2 \pi), \alpha \neq \beta$, the function $F_{0}(z)=\left\{\frac{f\left(e^{i \alpha} z\right)-f\left(z e^{i \beta}\right)}{e^{i \alpha}-e^{i \beta}}\right\} \varepsilon s_{\delta}^{*}$.

The proof of this lemma is contained in ([1], p. 68), hence, we omit it.

Lemma 2 and Theorem 3 imply that $F(z)$ defined in Theorem 8 belongs to $s_{m}^{*}$ where $m$ satisfies the inequality $m \leq(1-m) / 2(1+m)$. This proves Theorem 8 .

Similarly Lemma 2 and our Theorem 3 imply that F(z) defined by Theorem 1, of $\left([1]\right.$, $f \in K_{0}$ ) belongs to $S_{m}^{*}$ where $m$ is given by the inequality of Theorem 3 .

Acknowledgement. The author is thankful to Professor S.M. Shah for reading the manuscript and for helpful suggestions. 


\section{REFERENCES}

[1] BAJPAI, S.K. and Mehrok, T.J.S.: On Univalence of certain analytic functions associated with starlike, convex and close-to-convex functions, Indian J. Pure and App1. Math. 4 (1973), 66-72.

[2] BERNARDI, S.D.: Convex and starlike univalent functions, Trans. Amer. Math. Soc. 135 (1969), 429-446.

[3] CAUSEY, W.M. and White, W.L.: Starlikeness of certain functions with integral representations, J. Math. Anal. Appl. 64 (1978) 458-466.

[4] JACK, I.S.: Functions starlike and convex of order $\alpha$, J. London Math. Soc. $\underline{3}$ (1971), 469-474.

[5] LEWANDOWSKI, Z. Miller, S.S. and Zlotkiewicz, E.: Generating functions for some classes of univalent functions, Proc. Amer. Math. Soc. 56 (1976), 111-117.

[6] LIBERA, R.J.: Some Classes of regular univalent functions, Proc. Amer. Math. Soc. 16 (1965), 755-758.

[7] MILLER, S.S. Mocanu, P.T. and Reade, Maxwell 0.: Starlike integral operators, Pacific J. Math. $\underline{79}$ (1978), 157-168.

[8] MERKES, E.P. and Wright, D.J.: On the univalence of a certain integral, Proc. Amer. Math. Soc. 27 (1971), 97-100.

[9] MOCANU, P.T.: Une proprieté de convexité generalizée dans la representation conforme, Mathematica (Cluj) 11 (1969), 127-133.

[10] ROBERTSON, M.S.: An extremal problem for functions with positive real part, Mich. Math. J. 11 (1964), 327-335.

[11] SAKAGUCHI, K.: On certain univalent mappings, J. Math. Soc. Japan 11 (1959), 72-75.

[12] SILVIA, E.M.: On a subclass of spiral-like functions, Proc. Amer. Math. Soc. $44(1974), 411-420$.

[13] SINGH, R.: On Bazilevic functions, Proc. Amer. Math. Soc. 38 (1973), 261-271.

[14] SUFFRIDGE, T.J.: Some remarks on convex maps of the unit disk, Duke Math. J. $37(1970), 775-777$.

Current address: Department of Mathematics, Indiana - Purdue University at Fort Wayne

2101 Coliseum Boulevard East

Fort Wayne - INDIANA - 46805, USA 


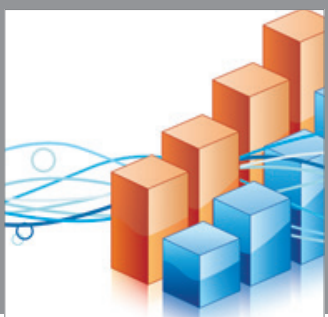

Advances in

Operations Research

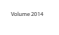

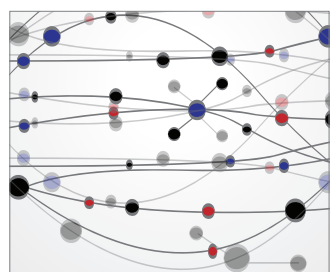

\section{The Scientific} World Journal
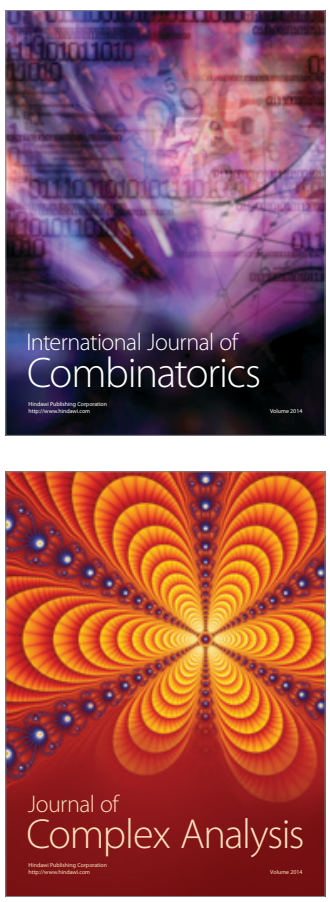

International Journal of

Mathematics and

Mathematical

Sciences
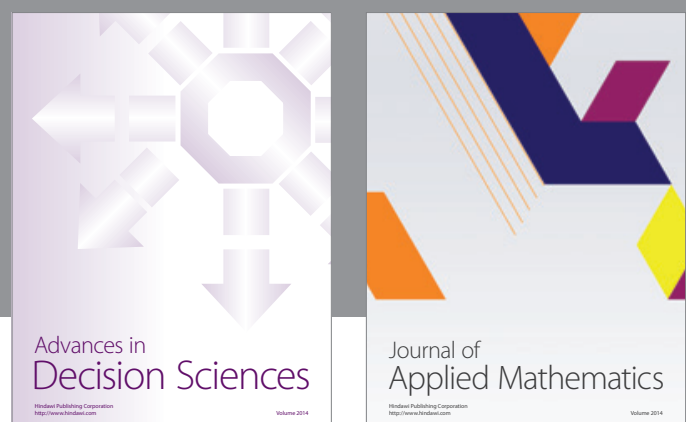

Journal of

Applied Mathematics
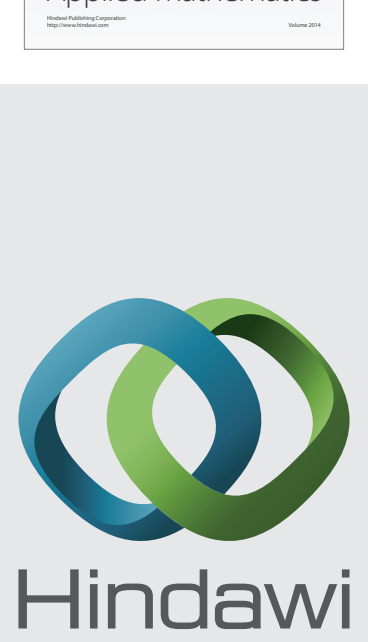

Submit your manuscripts at http://www.hindawi.com
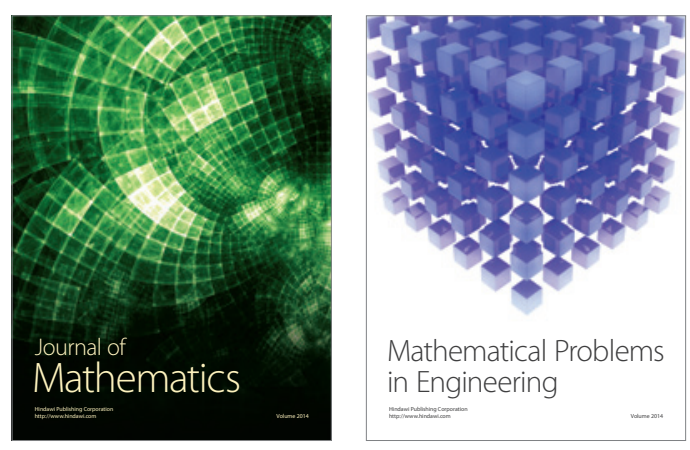

Mathematical Problems in Engineering
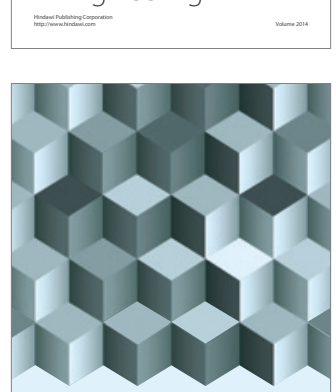

Journal of

Function Spaces
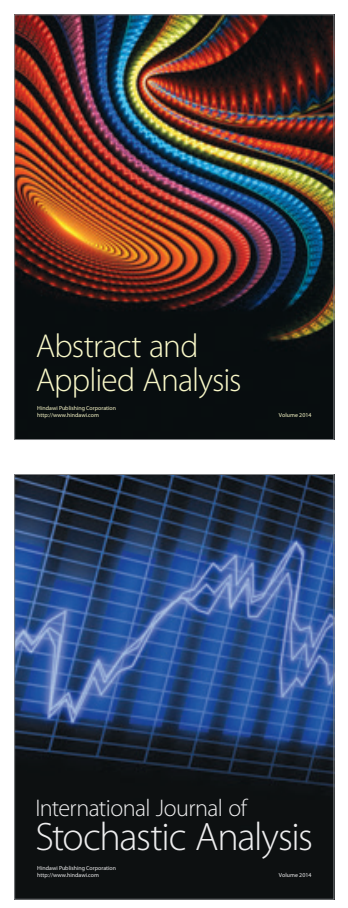

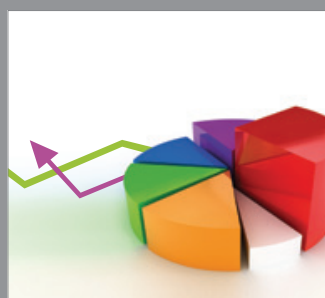

ournal of

Probability and Statistics

Promensencen
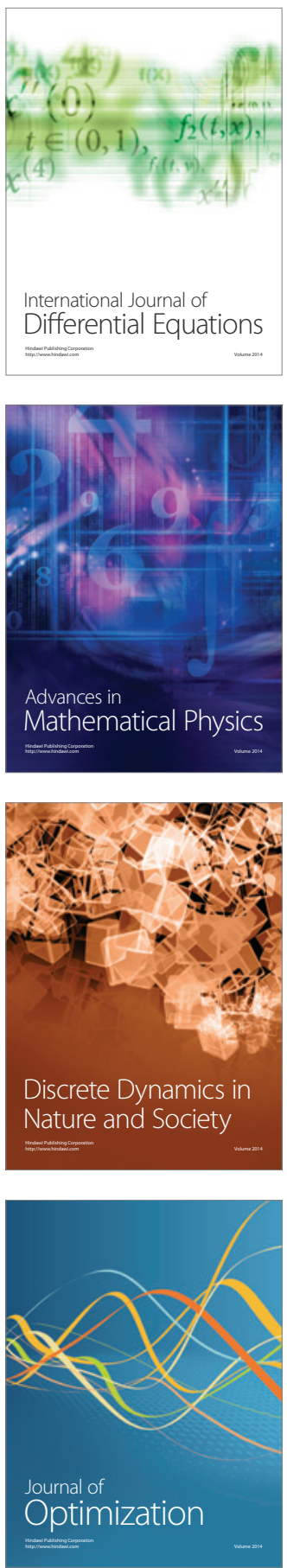